\title{
THE RANDOM WALK OF A SIMPLE RISK BUSINESS
}

\author{
H. L. SEAL \\ U.S.A.
}

\section{THE PROBLEM}

We suppose that a risk business issues a single type of contract under which, in return for a unit "premium", it will pay a "sum insured" $m$ ( $m$ being an integer) on the occurrence of a contingency of probability $q<\frac{1}{2}$. The expected gain on each contract is I- $m q$ and is assumed to be positive. This type of enterprise is conveniently denominated a simple risk business. Two "real life" situations it simulates are those of a group life policy for a uniform amount covering a number of young lives (e.g., university students), and a roulette casino where the stakes are uniform and the bets are limited to the single numbers o to 36 .

The risk business is supposed to commence its operations with a "risk reserve" of $K$ units. Each premium is added to this reserve as it is received and all claims by contract holders are paid therefrom. We say that the business is "ruined" as soon as the risk reserve becomes zero or negative (though it could be argued that it would be unethical to accept a premium once the risk reserve is less than $m-\mathrm{r}$ ). On the other hand, if the reserve reaches an amount $M$ units no further premiums are paid into it until a claim occurs to reduce it below $M$ (de Finetti, 1957). The risk business intends to continue its operations for a long, but finite, period unless ruined in the meantime.

We consider two probabilities: (i) $v_{x}$, the chance of eventual ruin given that the risk reserve is now $x$, and (ii) $v_{x}, n$, the probability that ruin occurs as a result of the $n$th contract (simultaneous contracts being ranked in a prearranged, e.g., alphabetical, order). Clearly

$$
v_{x}=\sum_{n=1}^{\infty} v_{x, n}
$$

and we will write $\mathrm{I}-v_{x}=u_{x}$. 


\section{HISTORY AND PRESENT STATUS}

The problem of a gambler with limited funds playing a series of games in each of which he has a constant probability of winning against an infinitely rich adversary is a special case of that in which the adversary has limited funds. The classical case assumes that the stake is a unit and the return is two units (including the player's own stake). The history of the calculation of $v_{x}$ and $v_{x}, n$ under these conditions is excellently sketched in Czuber (Igo6) and the mathematics are provided in $\S \S$ I-5 of ch. XIV of Feller (I957).

The more general problem in which the player's stake is $a$ units and the return is $a+b=m$ was first considered by Rouché (I888). He found the probability of a player with capital $A$ being ruined by an adversary with capital $B$ to be $v_{A}=\mathrm{I}-u_{A}$ where

$$
u_{A}=\left(\mathrm{I}-\lambda^{A}\right) /\left(\mathrm{I}-\lambda^{A+B}\right)
$$

and $\lambda$ is "la racine positive, autre que $I$ " of the equation

$$
p z^{a+b}-z^{a}+q=0 \text {. }
$$

Under these circumstances the expected duration of the game was found to be

$$
D_{A}=\left\{(A+B) u_{A}-A\right\} /\{(a+b) p-a\}
$$

where $p$ is the player's chance of winning any game. These results were reproduced by Bertrand (I889) in his text-book and similar, supposedly exact, formulae were derived by Bachelicr (I9r2) and Baudez (r947).

But in I903, in a Russian journal, Markov pointed out that equation (3) has $a+b$ roots and that they all contribute to the solution. By an elegant device, reproduced in his (IgI2) text-book, in Uspensky (I937), and briefly in Feller (I957), he obtained upper and lower bounds for $u_{x}(x=0, \mathrm{I}, 2, \ldots A+B)$. No attempt was made to obtain the value of $v_{x, n}$ for this general case.

During the second world war the problem was taken up once again in connection with the sequential sampling of manufactured items from a large batch. Wald's sequential procedure for accepting or rejecting the batch on the basis of the number of defective items in a growing sample can be expressed as a random walk along the $X$-axis commencing at a point $A$. One step is taken to the right along the axis whenever an acceptable item is inspected (with 
probability $p=\mathrm{I}-q$ ) and $a$ steps are taken to the left when a defective item is encountered (with probability q). Acceptance of the batch occurs with probability $u_{A}$ when the "absorbing barrier" $A+B$ is reached while attainment of, or passage through, the barrier at zero means that the batch should be rejected (the probability of rejection being $v_{A}$ ). It is readily seen that this procedure is equivalent to Rouché's problem with $b=\mathrm{I}$.

Burman (I946) and Girshick (I946) were able to obtain explicit expressions for $u_{x}$ under these conditions. Their answers appear as the quotient of two similar series. The former author also extended his procedure to the derivation of the generating function of $v_{x, n}$ (fixed $x$ ). From this he obtained expressions for the mean and variance of the random variable $N$, the number of items inspected, in terms of series of the same form as those appearing in $u_{x}$. Anscombe (I949) used these results to prepare tables of $m q$ (in our notation) and $E(N) / m$ for $u_{A}=.99, .9, .5$, . I and . or and a selected set of pairs of values of $A / m$ and $B / m$ ranging from (I, I) to $(i, j), i+j=8$. Finally, Walker (I950) generalized Burman's series results to include arbitrary integer $b$. Since the new formulae require the inversion of a matrix of order min $(a, b)$ they are not convenient for numerical application.

When we try to apply some of the foregoing results to the simple risk business defined above some unexpected difficulties arise. We now have $A=K, B \rightarrow \infty$, and $q$ possibly very small, e.g., .oor or less. The result is that $m$ is quite large and $K$ must be many times larger than $m$ if $v_{K}$, the risk of ultimate ruin, is to be very small. This in turn prevents the use of Burman's (I946) series for numerical calculations but encourages the employment of asymptotic results. In particular, we have been able to find simple approximate expressions for the mean and variance of the distribution of $N$ conditional on ruin occurring, and what appears to be a good approximation to the distribution itself by means of the Normal law.

\section{Determination of $\mathrm{v}_{x}$}

Following the arguments of Feller (r957) for the case $m=2$ it is observed that $u_{x}=\mathrm{I}-v_{x}$ satisfies the difference equation

$$
u_{x}=p u_{x+1}+q u_{x+1-m}(x=\mathrm{I}, 2, \ldots M-\mathrm{I})
$$


subject to the $m$ boundary conditions

$$
u_{x}= \begin{cases}0 & x=0,-\mathrm{I},-2, \ldots-(m-2) \\ u_{M} & x=M\end{cases}
$$

We note that $u_{M}$ will appear in the solution and must thus be given a numerical value. It represents the probability of not being ruined once the risk reserve has attained its ceiling of $M$. Suppose that the business is assumed to wind-up once it has issued $L$ further contracts after this point. Then $u_{M}$ decreases monotonically from unity, when $L<M /(m-\mathrm{x})$, to zero as $L \rightarrow \infty$. Thus a value of $L$ exists such that, with close approximation, $u_{M}$ equals any suitably chosen value, e.g., I-IO-8. In particular we may let $M \rightarrow \infty$ and write $\lim u_{M}=\mathrm{I}$.

$M \rightarrow \infty$

There are two commonly used methods of solving (5). The first is to note that the general solution has the form

$$
u_{x}=C_{1} \lambda_{1}^{x}+C_{2} \lambda_{2}^{x}+\ldots+C_{m} \lambda_{m}^{x}
$$

(as can be seen by substituting (6) into (5)) where $\lambda_{1}, \lambda_{2}, \ldots \lambda_{m}$ are the $m$ roots (supposed unequal) of the equation

$$
p z^{m}-z^{m-1}+q=0
$$

namely equation (3) with $a+b=m$ and $b=\mathrm{I}$.

Now (7) can be shown to have only two real positive roots $\lambda_{1}=\mathbf{I}$ and $\lambda_{2}=\lambda$, where $\lambda<\mathrm{I}$ provided $m q<\mathrm{I}$. [When $m q=\mathrm{I}$ there is a repeated root of unity, $u_{x}=0$ and ruin is certain. Ruin is also certain when $m q$, the expected claim, exceeds the unit premium.] When $m$ is odd there is one real negative root and when $m$ is even there are $m-2$ imaginary roots. All the $m-2$ roots that are not real and positive have moduli less than $\lambda$.

Hence, for large $x$,

$$
u_{x} \sim C_{1}+C_{2} \lambda^{x}
$$

and, using the boundary conditions at $x=0$ and $x=M$,

$$
u_{x} \sim \frac{\mathrm{I}-\lambda^{x}}{\mathrm{I}-\lambda^{m}} u_{M} \sim\left(\mathrm{I}-\lambda^{x}\right) u_{M}
$$

if $M$ is supposed very large in comparison with $x$. 
The Markov upper and lower bounds for the solution become in this case (cp. Uspensky, I937)

$$
\left(\mathrm{I}-\lambda^{x}\right) u_{M} \leq u_{x} \leq\left(\mathrm{I}-\lambda^{x+m-2}\right) u_{M}
$$

The alternative procedure for obtaining the solution of $(5)$ is to use the method of generating functions (Feller, I957; Ch. XI). It can be shown that in this case

$$
U(\theta) \equiv \sum_{x=1}^{M} u_{x} \theta^{x}=\frac{p u_{1} \theta^{1-m}}{q-\theta-m+1+p^{\theta-m}}
$$

Developing the right-hand side of this relation in powers of $\theta$ it is found that (cp. Burman, I946)

$$
u_{x}=\frac{H(x)}{H(M)} u_{M} \quad x=\mathrm{I}, 2, \ldots M
$$

where $\quad H(x) \equiv p^{-x+1} \sum_{k=0}^{\left[\frac{x-1}{m}\right]}(-\mathrm{I})^{k}\left(\begin{array}{c}x-\mathbf{I}-\overline{m-\mathbf{I}} k \\ k\end{array}\right)\left(p^{m-1} q\right)^{k}$ Alternatively we may express relation (I0) as the sum of $m$ partial fractions (Feller, I957) and obtain the asymptotic result

$u_{x} \sim\left\{\mathrm{I}-\frac{\mathrm{I}-m q}{m q \lambda^{1-m}-\mathrm{I}} \lambda x^{x} u_{M}\left\{\mathrm{I}-\frac{\mathrm{I}-m q}{m \lambda^{1-m}-\mathrm{I}} \lambda^{M}\right\}\right.$

in agreement with Cramér (I94I) when $M \rightarrow \infty$. It can be proved that the factor multiplying $\lambda^{x}$ in the expression on the right exceeds $\lambda^{m-2}$ but is less than unity. The result (I2) therefore lies between the two Markov bounds when $M \rightarrow \infty$.

To illustrate the foregoing formulae we may use parameters that correspond to the "real life" examples of our introductory paragraph.

Let us choose

(i) $m=900, q=.001, x=25,000$ and $u_{M}=\mathrm{I}$; and

(ii) $m=36, q=\mathrm{r} / 37, x=3,000$ and $u_{M}=\mathrm{I}$.

In case (i) we find $\lambda^{-1}=\mathrm{I} .00023044$ and (9) provides

$$
.99685 \leq u_{x} \leq .99744
$$

while (I2) becomes

$$
u_{x} \sim .99706
$$


On the other hand in illustration (ii) $\lambda=.998444$ and

$$
.99075 \leq u_{x} \leq .99123
$$

while (I2) gives

$$
u_{x} \sim .99095
$$

One is tempted to conclude that use of the left hand member of (9) will produce a value that is lower than the truth by not more than a few units in the fourth decimal place.

The CONTRACT $N$ at Which RUIN OCCURS

The probability that the random walk ends at the $n$th step after the commencement has been written $v_{x, n}$. By an argument similar to that used in obtaining the equation (5) we now have

$$
v_{x, n+1}=p v_{x+1, n}+q v_{x+1-m, n} \quad x=\mathrm{I}, 2, \ldots m-\mathrm{I}
$$

subject to the boundary conditions

and

$$
v_{x, n} \equiv 0, \quad x \leq 0, \quad n=\mathrm{I}, 2,3, \ldots
$$

$$
v_{x, n}=v_{M, n} \quad x=M+\mathrm{I}, M+2, \ldots
$$

We also adopt the convention that

$$
\begin{array}{ll}
v_{x, 0}=\mathrm{I} & x \leq 0 \text { (ruin has just occurred!) } \\
v_{x, 0}=0 & x=\mathrm{I}, 2,3, \ldots
\end{array}
$$

We now write the generating function of $v_{x, n}$ as

$$
V_{x}(\theta)=\sum_{n=0}^{\infty} v_{x, n} \theta^{n}
$$

multiply (13) by $\theta^{n+1}$ and sum over $n=0, \mathrm{I}, 2, \ldots$ The result is $V_{x}(\theta)=p \theta V_{x+1}(\theta)+q \theta V_{x+1-m}(\theta) \quad x=\mathrm{I}, 2,3, \ldots M-\mathrm{I} \quad$ (I9) We note that $V_{x}(\mathrm{I})=v_{x}$ from $(\mathrm{I})$.

Regarding $\theta$ as fixed at a value a little less than unity we may suppress it when used as an argument in (I9) and write

$$
V_{x}=p \theta V_{x+1}+q \theta V_{x+1-m} \quad x=\mathrm{I}, 2, \ldots M-\mathrm{I} \quad \text { (I9)' }
$$

with boundary conditions derived from (I3), (I4) and (I8), namely

$$
\begin{array}{ll}
V_{x}=v_{x, 0} \theta^{0}=\mathrm{I} & x=0,-\mathrm{I},-2, \ldots-(m-2) \\
V_{x}=v_{M}, n /(\mathrm{I}-\theta) & x=M
\end{array}
$$


This equation is similar to (5) but with different boundary conditions. Its characteristic equation

$$
p^{\theta} z^{m}-z^{m-1}+q \theta=0
$$

behaves in the same way as equation (7). In particular the two real roots $\lambda_{1}, \lambda_{2}$ have moduli in excess of those of the remaining $m-2$ roots. When $z=\mathrm{I}$ the left hand side of $(2 \mathrm{I})$ equals $\theta-\mathrm{I}$, which is negative by hypothesis, and thus $\lambda_{1}>\mathrm{I}$ and $\lambda_{2}<\mathrm{I}$.

Hence

$$
V_{x}(\theta) \sim C_{1}(\theta)\left\{\lambda_{1}(\theta)\right\}^{x}+C_{2}(\theta)\left\{\lambda_{2}(\theta)\right\}^{x}
$$

where dependency on $\theta$ has now been made explicit, and the procedure that previously led to the Markov upper and lower bounds (Uspensky, I937) now results in

$$
\left\{\lambda_{2}(\theta)\right\}^{x+m-2} \leq V_{x}(\theta) \leq\left\{\lambda_{2}(\theta)\right\}^{x}
$$

which is equivalent to (9) (with $u_{M}=\mathrm{I}$ ) when $\theta=\mathrm{I}$. We must therefore find an approximation to $\lambda_{2}(\theta)$.

When $\theta=\mathrm{I}$ equation ( $2 \mathrm{I}$ ) reduces to equation (7) which has a root $\lambda<\mathrm{I}$. We therefore write

$$
\lambda_{2}(\theta)=\lambda+\frac{\varepsilon(\theta)}{m}
$$

where $\varepsilon(\theta)$ is to be determined. Suppressing the $\theta$ of $\varepsilon(\theta)$ equation (2I) may now be written

$$
p \theta \lambda^{m}\left(\mathrm{I}+\frac{\varepsilon}{\lambda m}\right)^{m}-\lambda^{m-1}\left(\mathrm{I}+\frac{\varepsilon}{\lambda m}\right)^{m-1}+q \theta=0
$$

or, approximately (since $m$ is large),

$$
p \theta \lambda^{m} e^{\varepsilon / \lambda}-\lambda^{m-1} e^{\varepsilon / \lambda}+q \theta=0
$$

so that

$$
e^{\varepsilon / \lambda} \cong q \theta /\left(\lambda^{m-1}-p^{\theta} \lambda^{m}\right)
$$

and from (24)

$$
\lambda_{2}(\theta) \cong \lambda\left\{\mathbf{I}+m^{-1} \ln \left[q \theta /\left(\lambda^{m-1}-p \theta \lambda^{m}\right)\right]\right\}
$$

where $\lambda$ is the real positive root of equation $(7)$ that is less than unity. 
Hence

$$
\begin{gathered}
V_{x}(\theta) \sim \lambda^{x}\left\{\mathrm{I}+m^{-1} \ln \left[q \theta /\left(\lambda^{m-1}-p \theta \lambda^{m}\right)\right]\right\}^{x} \\
\sim V_{x}(\mathrm{I}) M_{x}(\tau)=v_{x} M_{x}(\tau)
\end{gathered}
$$

where $\theta=e^{\tau}$ and

$$
M_{x}(\tau)=\left\{\mathrm{I}+m^{-1} \ln \left[q e^{\tau} /\left(\lambda^{m-1}-p \lambda^{m} e^{\tau}\right)\right]\right\}^{x}
$$

is the moment generating function of the random variable $N$, the number of contracts until ruin occurs ${ }^{1}$ ), given that ruin is certain with a current risk reserve of $x$ units.

Now

$$
M_{1}(\tau)=\mathrm{I}+m^{-1} \ln \left(q / \lambda^{m-1}\right)+m^{-1} \tau-m^{-1} \ln \left(\mathbf{I}-p \lambda e^{\tau}\right)
$$

and

$$
\begin{aligned}
& \ln \left(\mathrm{I}-p \lambda e^{\tau}\right)=-\sum_{j=1}^{\infty} \frac{\mathrm{I}}{j}(p \lambda)^{j} \sum_{k=0}^{\infty} \frac{(j \tau)^{k}}{k !} \text { provided }\left|p \lambda e^{\tau}\right|<\mathrm{I} \\
& =-\sum_{k=0}^{\infty} \frac{\tau^{k}}{k !} \sum_{j=1}^{\infty} j^{k-1}(p \lambda)^{j} \\
& =\ln (\mathrm{I}-p \lambda)-p \lambda(\mathrm{I}-p \lambda)^{-1} \tau-\sum_{k=2}^{\infty} \frac{\tau^{k}}{k !} \sum_{j=0}^{\infty}(p \lambda)^{j} \sum_{i=1}^{k-1} s_{k-1}^{l} j^{(l)}
\end{aligned}
$$

where $s_{k-1}^{l}$ is a Stirling number of the second kind (Buckingham, I957)

$$
=\ln (\mathrm{I}-p \lambda)-p \lambda(\mathrm{x}-p \lambda)^{-1} \tau-\sum_{k=2}^{\infty} \frac{\tau^{k}}{k !} \sum_{l=1}^{k-1} s_{k-1}^{l} l !(p \lambda)^{l} /(\mathrm{I}-p \lambda)^{l+1}
$$

We may thus calculate the cumulants of $N$ with $x=\mathrm{I}$ up to any desired order (Kendall \& Stuart, I958) and, on multiplication by $x$, the corresponding cumulants of $N$ with $x$ an integer greater than unity.

In fact it can be shown that the mean and variance of this distribution of $N$ are

$$
x m^{-1}(\mathbf{I}-p \lambda)^{-1} \text { and } x m^{-2}(m p \lambda-\mathrm{I})(\mathbf{I}-p \lambda)^{-2},
$$

1) i.e., the number of steps to first-passage of the barrier at zero. 
respectively. Furthermore the Fisher measures of skewness and kurtosis tend asymptotically to

$$
\frac{\mathrm{I}-p \lambda}{(p \lambda)^{2}}\left(\frac{m}{x}\right) \text { and } \frac{\mathrm{I}+4 p \lambda+(p \lambda)^{2}}{p \lambda} \cdot \frac{m}{x}
$$

respectively. Since $p \lambda$ is close to unity both these parameters converge rapidly towards zero as $x$ increases.

Reverting to the two numerical illustrations used earlier the foregoing parameters become:

\begin{tabular}{|c|c|c|c|}
\hline $\begin{array}{l}\text { mean } \\
\text { skewness }\end{array}$ & $\begin{array}{l}=22,580.7 \\
\sim .0002\end{array}$ & $\begin{array}{l}\text { variance } \\
\text { kurtosis }\end{array}$ & $\begin{array}{l}=(4279.364)^{2} \\
\sim .2160\end{array}$ \\
\hline mean & $=2,9 \mathrm{I} 9.78$ & variance & $=(310.7086)^{2}$ \\
\hline skewness & $\sim .0033$ & kurtosis & $\sim .0720$ \\
\hline
\end{tabular}

An indication of the corresponding conditional distributions of risk is given by the following table.

Probability that if ruin is certain it occurs at or before contract $n$
Approximate value of $n$ for illustration:

(i)

\begin{tabular}{lll}
\hline I & $\mathrm{I} 7,096$ & $2,52 \mathrm{I}$ \\
.2 & 18,979 & 2,658 \\
.3 & 20,336 & 2,756 \\
.4 & $2 \mathrm{I}, 496$ & $2,84 \mathrm{I}$ \\
.5 & 22,580 & $2,9 \mathrm{I} 9$ \\
.6 & 23,664 & 2,998 \\
.7 & 24,824 & 3,082 \\
.8 & $26, \mathrm{I} 82$ & $3, \mathrm{I} 8 \mathrm{I}$ \\
.9 & 28,064 & $3,3 \mathrm{I} 7$ \\
\hline
\end{tabular}

\section{REFERENCES}

Anscombe, F. J. (I949) Tables of sequential inspection schemes to control fraction defective. J.R. Statist. Soc., $A$, II2, I80-206.

Bachelier, L. (I9I2) Calcul des Probabilités. Paris.

Baudez, G. (1947) Le plein dans les compagnies d'assurances. Bull. Trimest. Inst. Actu. Franç. 50-54, I3-88.

Bertrand, J. (1889) Calcul des Probabilités. Paris.

Burman, J. P. (1946) Sequential sampling formulae for a binomial population. J. R. Statist. Soc., B, 8, 98-103.

Buckingham, R. A. (I957) Numerical Methods. Pitman, London.

Cramér, H. (I94I) Deux conférences sur la théorie des probabilités. Skand. Aktuartidskr. 24, 34-69. 
Czuber, E. (Igo6) Calcul des probabilités. Jules Molk, Ed., Encyclopédie des Sciences Mathématiques, Tome I, Vol. 4, Fasc. I, Paris.

Feller, W. (I957) An Introduction to Probability Theory and Its Applications. Wiley, New York.

FinetTi, B. DE (I957) Su un'impostazione alternativa della teoria collettiva del rischio. Trans. $X V$ Intern. Cong. Actu., New York, 2, 433-44I.

Girshick, M. A. (1946) Contributions to the theory of sequential analysis, II, III. Ann. Math. Statist., I 7, 282-298.

Kendall, M. G. \& Stuart, A. (1958) The Advanced Theory of Statistics. Griffin, London.

MARKOFF, A. A. (1912) Wahrscheinlichkeitsrechnung. Leipzig-Berlin.

Rouchê, E. (I 888) Sur un problème relatif à la duréo du jeu. C. R. Acad. Sci. Paris, II $6,47-49$.

Uspensky, J. V. (I937) Introduction to Mathematical Probability. Mc(iraw Hill, New York.

Walker, A. M. (I950) Note on sequential sampling formulae for a binomial population. I. R. Statist. Soc., B, I 2, 301-307. 